\title{
In Liguria le cozze scalzano i muscoli
}

\author{
Vittorio Coletti
}

PUBBLICATO: 29 LUGLIO 2016

\section{Quesito:}

Ci sono giunte dalla Liguria varie segnalazioni sulla progressiva sostituzione del termine ittico locale muscolo con quello meridionale cozza; anche da altre zone ci si chiede se non sia meglio usare cozze, comprensibile in tutta Italia, invece del tradizionale, ma poco diffuso mitilo.

\section{In Liguria le cozze scalzano i muscoli}

I

n Liguria è scoppiato il caso dei muscoli scalzati dalle cozze in una pescheria per iniziativa, pare, della Guardia di Finanza. Di qui proteste come quelle del signor Falcone che ci danno modo di rispondere anche ad altri che hanno chiesto qual è il nome dei... mitili che mangiamo spesso alla marinara o al pomodoro o con la pasta.

Cominciamo col dire che i mitili (questo il loro nome scientifico cui si affianca anche quello meno specialistico didatteri di mare), molluschi bivalvi lamellibranchi della famiglia del Mitilidi, cosi nominati già nel Quattrocento, sono noti nell'Italia centrale e nordoccidentale col nome di muscoli, secondo una trafila che discende direttamente dal latino (anche se non si sa se il musculus mitilo sia davvero la stessa parola del musculus muscolo) e con questo nome sono attestati da scrittori settentrionali e toscani a partire dal Cinque-Seicento. Dall'inizio del Novecento sono però sempre più conosciuti in Italia col nome di origine meridionale cozze, esito locale di coccia, che, diversamente da quanto ipotizzato da un lettore, è un derivato di cochleam, che significava chiocciola (e in seguito anche testa) e quindi nominava efficacemente l'animale dal guscio, dalle valve. Nell'Italia nordorientale e soprattutto nel veneziano gli stessi mitili sono chiamati peoci (forma veneta del termine pidocchi, come a dire 'pidocchi di mare').

Si tratta dunque di un tipico caso di geosinonimi che nominano in modo diverso in diverse regioni una stessa cosa, quella che nella lingua scientifica è appunto chiamata mitilo. Qual è il nome più giusto? Duole deludere qualche tifoso del nome di casa, ma nessuno è sbagliato. Se si chiede qual sarebbe il più funzionale in italiano si potrà osservare che: muscolo è di attestazione più antica e il suo etimo ha diffusione europea in questo significato, come giustamente è stato notato (francese, tedesco ecc.). Quanto a albero genealogico, muscolo meriterebbe dunque il primo posto, così come peocio va sicuramente all'ultimo per la sua circolazione solo locale. Cozza è di attestazione nazionale più recente ma di grande successo. Poiché la decisione non la prende né l'amatore della lingua di casa né il finanziere né il linguista, ma l'uso, vediamo con l'aiuto di Google come si sta comportando l'uso, tenendo comunque presente che nella vigente Denominazione in lingua italiana delle specie ittiche di interesse commerciale (Decreto del Ministero delle Politiche Agricole Alimentari e Forestali del 3I gennaio 2008, G.U. n. 45 del 22 febbraio 2008, e successive integrazioni fino al decreto I2 agosto 20II, G.U. n. 208 del 7 settembre 20II) troviamo "Cozza o Mitilo" in corrispondenza del nome latino Mytilus galloprovincialis, mentre non c'è traccia di muscolo (né di peocio). E dunque se cerchiamo "cozze" e "muscoli alla" (marinara ecc.) vediamo che le prime sono attestate quasi dieci volte di più dei secondi e che le "cozze al" (pomodoro ecc.) sono cinque volte più frequenti dei "muscoli al". Stesso risultato se cerchiamo "insalata o zuppa di cozze" o "di muscoli". 
Che concluderne? Poiché, come si diceva, la lingua va dove l'uso vuole, temo per i miei corregionali liguri, particolarmente dispiaciuti per le sfortune dei muscoli cosi ben cucinati nello spezzino (e comunque ancora molto solidi nell'italiano locale), che le cose volgano nella lingua nazionale a favore delle cozze, anche senza bisogno di troppo zelanti guardie di finanza. Sarebbe semmai interessante chiedersi perché il geosinonimo meridionale scalzi quello centrosettentrionale. Forse perché la cucina di pesce meridionale ha più successo di quella delle altre regioni?

\section{Cita come:}

Vittorio Coletti, In Liguria le cozze scalzano imuscoli , "Italiano digitale", 2016, IX, 2019/2 (aprilegiugno)

DOI: $10.35948 / 2532-9006 / 2020.3156$

Copyright 2016 Accademia della Crusca

Pubblicato con licenza creative commons CC BY-NC-ND 